\title{
Infrared and radio study of the W43 cluster
}

\section{Resolved binaries and non-thermal emission}

\author{
P. L. Luque-Escamilla ${ }^{1,3}$, A. J. Muñoz-Arjonilla ${ }^{2,3}$, J. R. Sánchez-Sutil ${ }^{3}$, J. Martî2,3, \\ J. A. Combi ${ }^{4}$, and E. Sánchez-Ayaso ${ }^{2,3}$
}

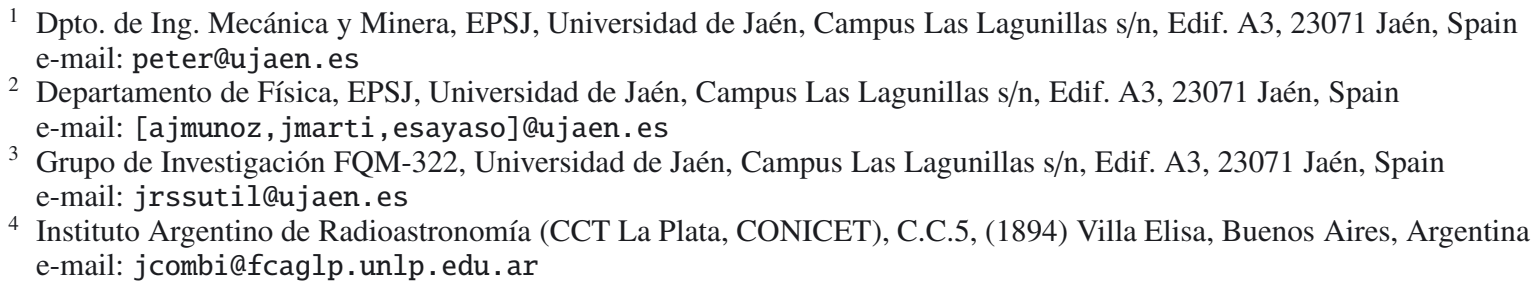

Received 20 May 2011 / Accepted 1 July 2011

\section{ABSTRACT}

\begin{abstract}
Context. The recent detection of very high-energy (VHE) gamma-ray emission from the direction of the W43 star-forming region prompted us to investigate its stellar population in detail in an attempt to see wether or not it is possible an association.

Aims. We search for the possible counterpart(s) of the gamma-ray source or any hints of them, such as non-thermal synchrotron emission as a tracer of relativistic particles often involved in plausible physical scenarios for VHE emission.

Methods. We data-mined several archival databases with different degrees of success. The most significant results came from radio and near-infrared archival data.

Results. The previously known Wolf-Rayet star in the W43 central cluster and another cluster member appear to be resolved into two components,suggesting a likely binary nature. In addition, extended radio emission with a clearly negative spectral index is detected in coincidence with the W43 cluster. These findings could have important implications for possible gamma-ray emitting scenarios, which we also briefly discuss.
\end{abstract}

Key words. stars: Wolf-Rayet - X-rays: stars - radio continuum: stars - gamma rays: stars - infrared: stars

\section{Introduction}

W43 (=G30.8-0.2) is a star-forming complex first detected at radio wavelengths by Westerhout (1958). Recently, attention has been focused on it, because it is believed to be one of the closest starburst regions in our galaxy (see Bally et al. 2010, and references therein). The distance to W43 was originally estimated based on HI absorption and reddening measurements (Smith et al. 1978; Blum et al. 1999) pointing to a $5.5 \mathrm{kpc}$ value, although with a significant uncertainty. An improved estimate using $6.7 \mathrm{GHz}$ methanol masers yielded a more reliable average systemic velocity of $97.5 \mathrm{~km} \mathrm{~s}^{-1}$, corresponding to a larger distance of $9.0 \pm 0.4 \mathrm{kpc}$ (Pandian et al. 2008). A recent revision of survey molecular emission from W43 confirms a distance consistent with the 6 to $9 \mathrm{kpc}$ range, with preference toward the lower limit, and a total mass of about $7 \times 10^{6} M_{\odot}$ (Nguyen Luong et al. 2011). We also refer the reader to this work for a wide-field view of the W43 as part of a very extended radio source in the galactic plane. From the cluster color-color diagram, Blum et al. (1999) derived a considerable extinction value of $A_{\mathrm{V}} \simeq 34 \mathrm{mag}$, equivalent to a hydrogen column density of about $6.5 \times 10^{22} \mathrm{~cm}^{-2}$.

The GLIMPSE image displayed in Fig. 1 shows the W43 field in the infrared band. Among other components, W43 contains a central open cluster of massive stars illuminating a giant HII region, which will be better displayed below. A decade ago, Blum et al. (1999) studied the W43 cluster in detail showing that its three brightest members include one Wolf-Rayet (WR) and two luminous O-type stars. These objects are currently designated as W43 \#1, W43 \#2 and W43 \#3, respectively. The same authors classified the first of them as of WN7+abs spectral type and suggested that the presence of absorption features could be related to an unresolved companion. W43 \#1 was subsequently included in the 7th Catalogue of Galactic Wolf-Rayet stars (van der Hutch 2001), where it is also listed as WR 121a.

The W43 cluster is remarkably coincident in position with a VHE gamma-ray source detected by the H.E.S.S. collaboration at TeV energies (Chaves et al. 2009). This VHE object is known as HESS J1848-018 and it appears to be clearly extended with respect to the point-spread function of this Imaging Atmospheric Cherenkov Telescope array. If the association with the cluster is correct, this will be the second proposed case of gamma-ray emission from a stellar cluster after the well known Westerlund 2 (Aharonian et al. 2007). W43 \#1 (=WR 121a) has been also considered by the HESS J1848-018 discoverers as a possible counterpart candidate. Another gamma-ray source detected by the Fermi Large Area Telescope (LAT) is also present in the field (Abdo et al. 2009, 2010). However, its latest available LAT error box corresponding to the 1FGL J1848.1-0145c entry in the Fermi catalog excludes the W43 position at present. Whether the HESS source is connected with the Fermi GeV emission or with the W43 stellar cluster still remains to be confirmed. Fermi 


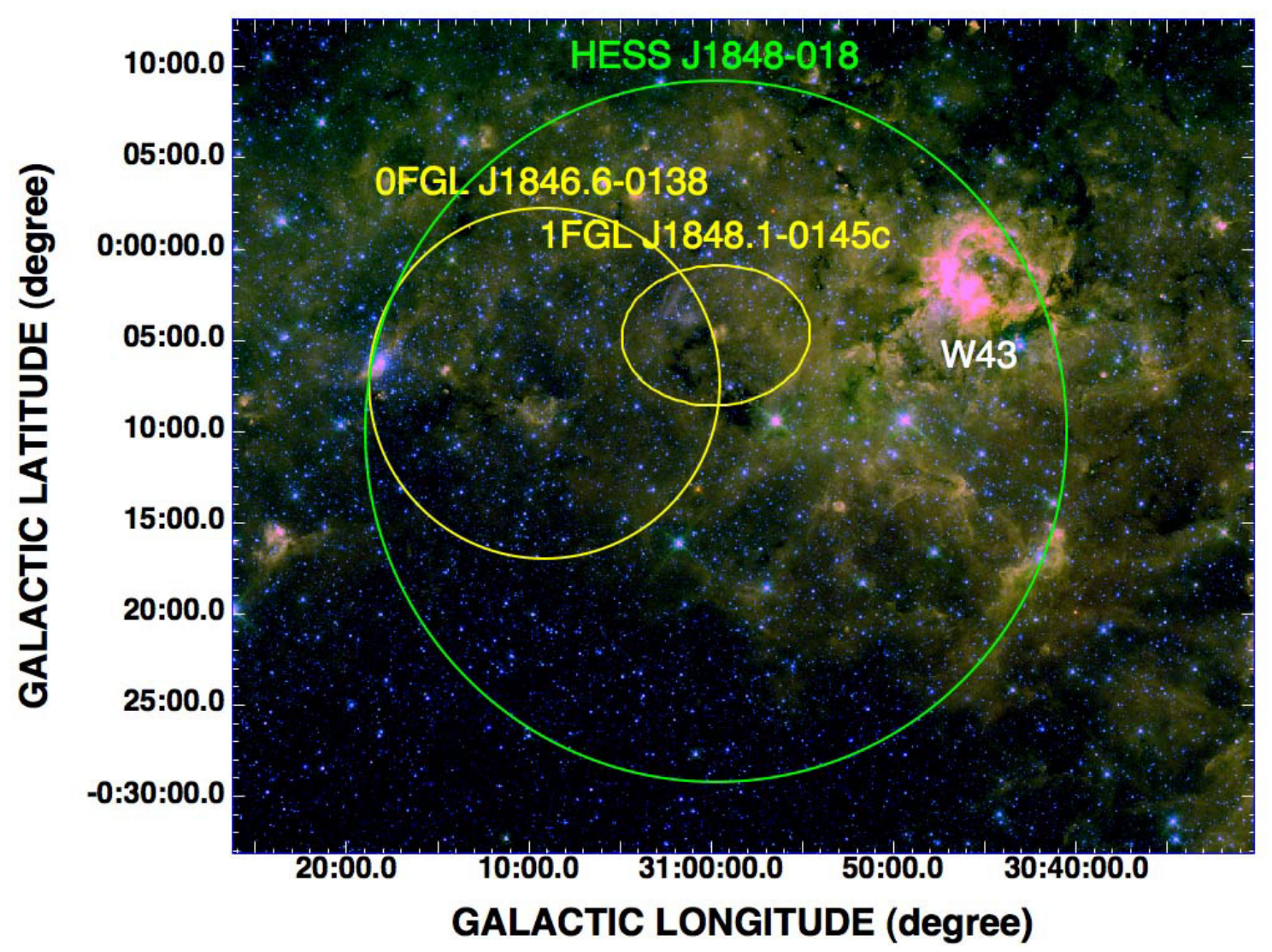

Fig. 1. Composite trichromatic image of the W43 cluster the GLIMPSE 3.6, 5.8 and $8 \mu \mathrm{m}$ bands in blue, green and red color, respectively. The green and yellow ellipses indicate the positions of the HESS and Fermi LAT sources in the field. The W43 star-forming region, consistent with the HESS position, is also indicated with a white label.

has a strong background uncertainty in this region, which could imply significant position shifts in future improved versions of its catalog.

In this context, we report here new observational results about W43 mostly based on archival data. First, we present nearinfrared observations that provide strong evidence for the binary nature of the cluster WR star and another likely member as well. Secondly, we present the detection of extended non-thermal radio emission apparently coming from the cluster itself. Both could have strong implications in our understanding of possible gamma-ray emitting scenarios, and the third part of this paper is devoted to discuss this briefly.

\section{Analysis of ESO archive infrared observations}

We retrieved different observing epochs of W43 from the European Southern Observatory (ESO) archives. The position of W43 was covered several times with the UT1 unit of the ESO Very Large Telescope (VLT) and its ISAAC instrument. In particular, we downloaded archive images obtained the on 2001 July 10 in the $J$ - and $K$ s-bands under especially good seeing conditions (0.'4). Data reduction was performed by means of the IRAF package mainly including flat-field, sky-background subtraction and median combining of individual frames. An astrometric solution was finally computed for the final images using between 20 and 30 unsaturated stars. Their reference positions were taken from the Two Micron All Sky Survey (2MASS, Skrutskie et al. 2006). The residuals of the astrometric fit had an rms value of $\pm 0{ }^{\prime} 09$ and \pm 0 .' 10 in right ascension and declination, respectively. In Fig. 2 we show the resulting composite-color image for the W43 cluster together with radio contours that will be discussed below. The angular resolution of this VLT infrared image fairly exceeds those previously published thanks to the excellent quality of the seeing. This circumstance allows us to clearly distinguish the binary nature of the WR star W43 \#1 as seen in the enlarged $J$-band view shown in Fig. 3. Hereafter, the individual components of this system will be referred to as W43 \#1a and W43 \#1b. They are separated by $598 \pm 3$ mas with position angle of $255^{\circ} \pm 1^{\circ}$. In addition, we also detected another likely faint companion to star W43 \#3 (see Fig. 4 with proposed components labeled W43 \#3a and W43 \#3b). Their angular separation is $640 \pm 100$ mas with position angle of $271^{\circ} \pm 7^{\circ}$.

The 2MASS stars were also used to provide a zero-point photometric calibration and derive the magnitudes listed in Table 1 for the apparent binaries quoted above together with their positions. Typical errors here are \pm 0 .' 1 for each coordinate and \pm 0.1 mag for the photometry, which was especially difficult because of the proximity of each star pair. Magnitudes not listed in Table 1 correspond to saturated or undetected stars.

\section{Analysis of the NRAO archive radio observations}

Several sets of radio observations obtained with the Very Large Array (VLA) of the National Radio Astronomy Observatory (NRAO) in the USA were used in this work. They were either retrieved from public surveys such as MAGPIS (Helfand et al. 2006), or directly downloaded and calibrated from the NRAO public archives. The AIPS software package and standard interferometer calibration techniques were used for this purpose. 


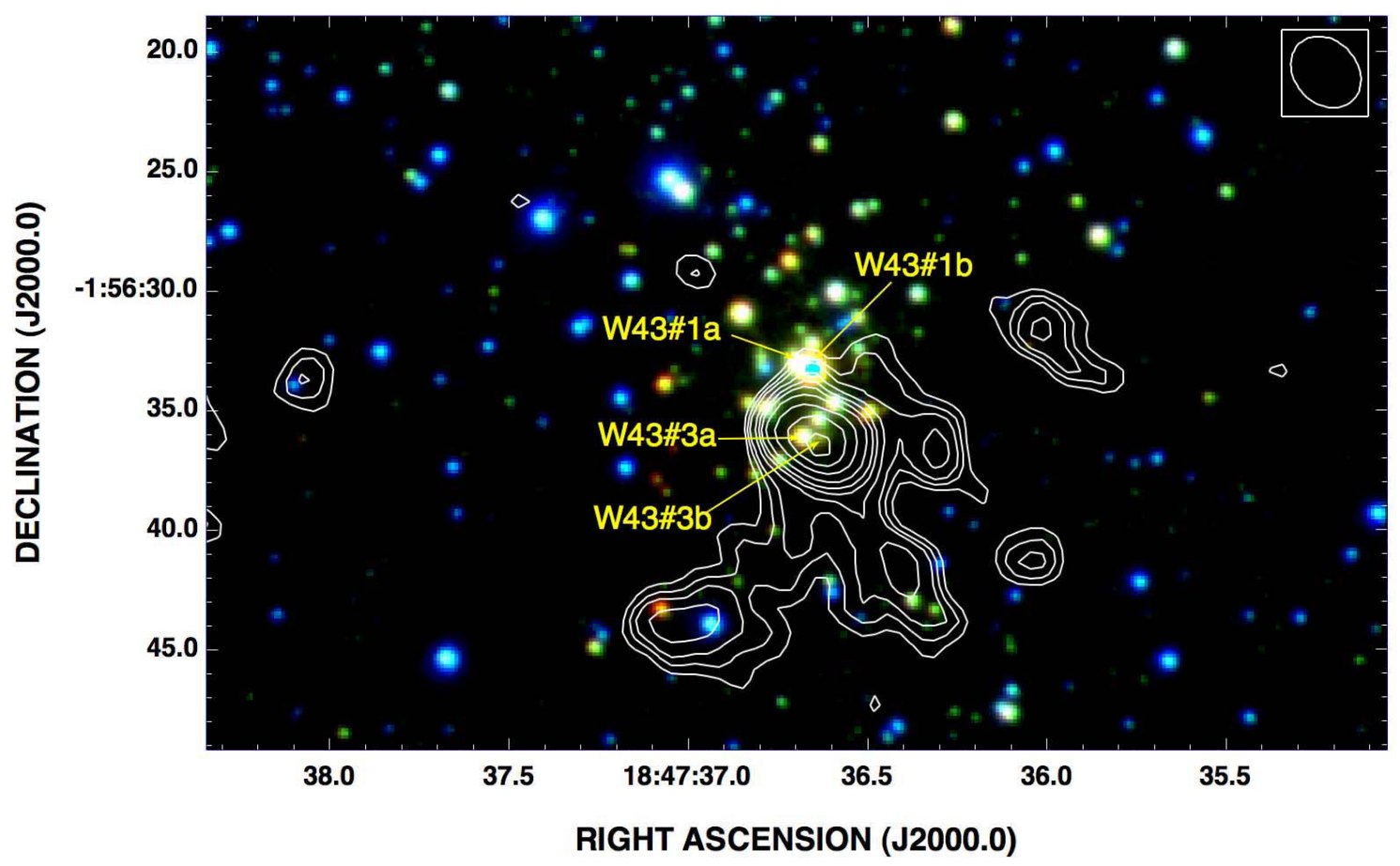

Fig. 2. Composite trichromatic image of the W43 cluster with the ESO VLT $J, H$ and $K$ s bands in blue, green and red color, respectively. The positions of the W43 \#1 and W43 \#3 stars proposed binary components are indicated by arrows. White contours correspond to $6 \mathrm{~cm}$ radio emission as observed with the VLA in its extended B-configuration. Contours shown correspond to 4, 5, 6, 8, 10,12, 15, 20, 25 and 30 times the rms noise of $0.76 \mathrm{mJy} \mathrm{beam}^{-1}$. The VLA synthesized beam is plotted as an ellipse at the top right corner and corresponds to $11^{\prime \prime} 63 \times 11^{\prime \prime} 31$, with position angle of $42^{\circ} .45$.

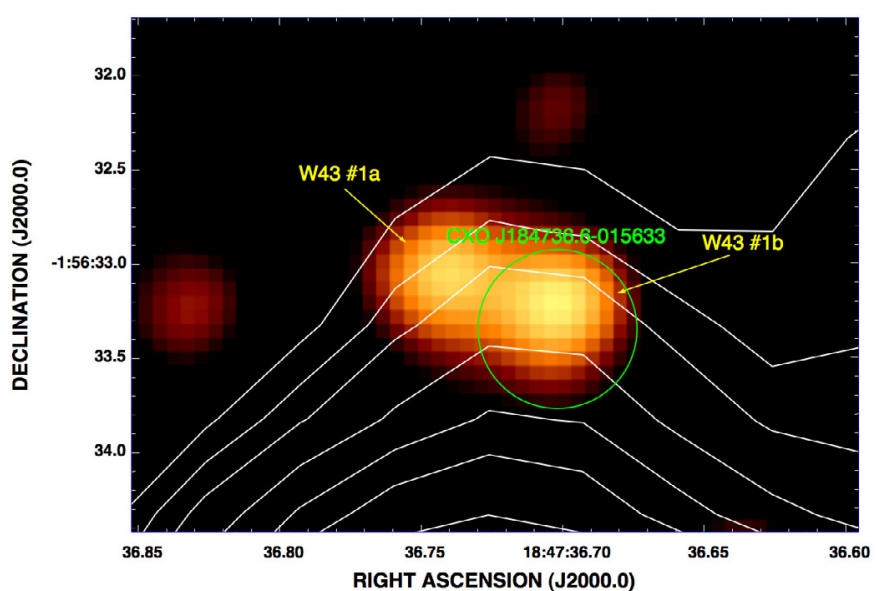

Fig. 3. Zoomed $J$-band view of the WR star W43 \#1 with its components $\mathrm{a}$ and $\mathrm{b}$ clearly resolved. The green circle represents the CXO J184736.6-015633 position at the $95 \%$ confidence level according to the Chandra X-ray source catalog. Radio contours are the same as in Fig. 2.

Table 2 lists the details of VLA observations together with the flux densities measured for the radio source detected in the direction of the W43 central cluster. This object has a clear extended morphology at all wavelengths, and we include a contour plot of our best high angular resolution map at the $6 \mathrm{~cm}$ wavelength in Fig. 2. Part of these contours also appear in Figs. 3 and 4. The central condensation of the radio emission has a deconvolved angular size of $\left(33^{\prime \prime} 8 \pm 0 .{ }^{\prime} 1\right) \times\left(3{ }^{\prime \prime} 1 \pm 0.1\right)$, with position angle of $61^{\circ} \pm 8^{\circ}$. The $\mathrm{J} 2000.0$ position of the radio peak is centered at $18^{\mathrm{h}} 47^{\mathrm{m}} 36^{\mathrm{s}} .63 \pm 0.01$ and $-01^{\circ} 56^{\prime} 36^{\prime \prime} \cdot 3 \pm 00^{\prime} 1$.

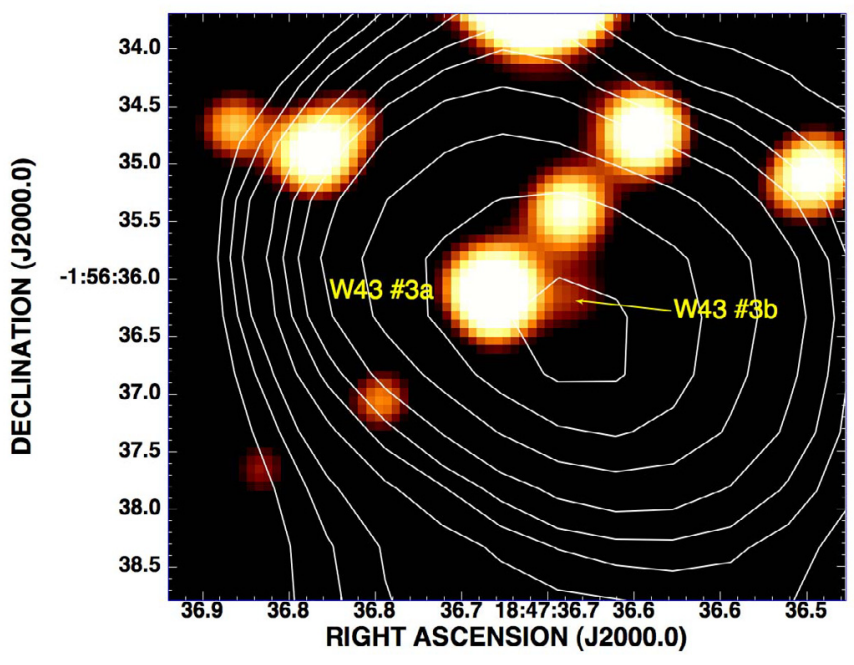

Fig. 4. Zoomed $K$ s-band view of the O-type star W43 \#3 with its proposed faint companion shown by an arrow. Radio contours are the same as in Fig. 2.

Besides obtaining a high angular resolution map, data sets in Table 2 were selected to ensure nearly matching-beam observations at three different wavelengths. Having similar synthesized beams at all wavelengths is the best approach to measure reliable spectral indices for any object in the field. The radio source coincident with the W43 cluster appears to be clearly non-thermal, as shown in the Fig. 5 spectrum. A simple power-law fit indicates that the observed radio emission is well represented by $S_{v}=(0.48 \pm 0.02 \mathrm{Jy})[v / \mathrm{GHz}]^{-0.47 \pm 0.04}$. The total radio luminosity integrated from 0.1 to $100 \mathrm{GHz}$ amounts to $1.0 \times 10^{33} \mathrm{erg} \mathrm{s}^{-1}$ with a brightness temperature as high as $7.1 \times 10^{4} \mathrm{~K}$. 
Table 1. Observed near-infrared positions and magnitudes.

\begin{tabular}{lccccc}
\hline \hline Object & $\alpha_{\text {J2000.0 }}$ & $\delta_{J 2000.0}$ & $J$ & $H$ & $K \mathrm{~s}$ \\
\hline W43 \#1a & $18^{\mathrm{h}} 47^{\mathrm{m}} 36^{\mathrm{s}} .691$ & $-01^{\circ} 56^{\prime} 33^{\prime \prime} 06$ & 16.1 & - & - \\
W43 \#1b & $18^{\mathrm{h}} 47^{\mathrm{m}} 36.653$ & $-01^{\circ} 56^{\prime} 33^{\prime \prime} 22$ & 15.8 & - & - \\
\hline W43 \# 3a & $18^{\mathrm{h}} 47^{\mathrm{m}} 36.677$ & $-01^{\circ} 56^{\prime} 36^{\prime \prime} 11$ & 17.6 & 13.5 & 11.5 \\
W43 \# 3b & $18^{\mathrm{h}} 47^{\mathrm{m}} 36.648$ & $-01^{\circ} 56^{\prime} 36^{\prime \prime} 08$ & - & - & 13.6 \\
\hline
\end{tabular}

Table 2. Radio emission from the W43 central cluster.

\begin{tabular}{lcccc}
\hline \hline $\begin{array}{l}\text { Data } \\
\text { origin }\end{array}$ & $\begin{array}{c}\text { Observation } \\
\text { epoch }\end{array}$ & $\begin{array}{c}\text { VLA } \\
\text { Conf. }\end{array}$ & $\begin{array}{c}\text { Frequency } \\
(\mathrm{GHz})\end{array}$ & $\begin{array}{c}\text { Flux density } \\
(\mathrm{Jy})\end{array}$ \\
\hline MAGPIS & 2007 Apr. 25 & $\mathrm{B}$ & 1.465 & $0.40 \pm 0.03$ \\
AD129 & 1984 May 03 & $\mathrm{C}$ & 4.860 & $0.25 \pm 0.03$ \\
& 1985 May 17 & $\mathrm{B}$ & 4.866 & - \\
AM663 & 2000 Jul. 26 & D & 8.460 & $0.17 \pm 0.02$ \\
\hline
\end{tabular}

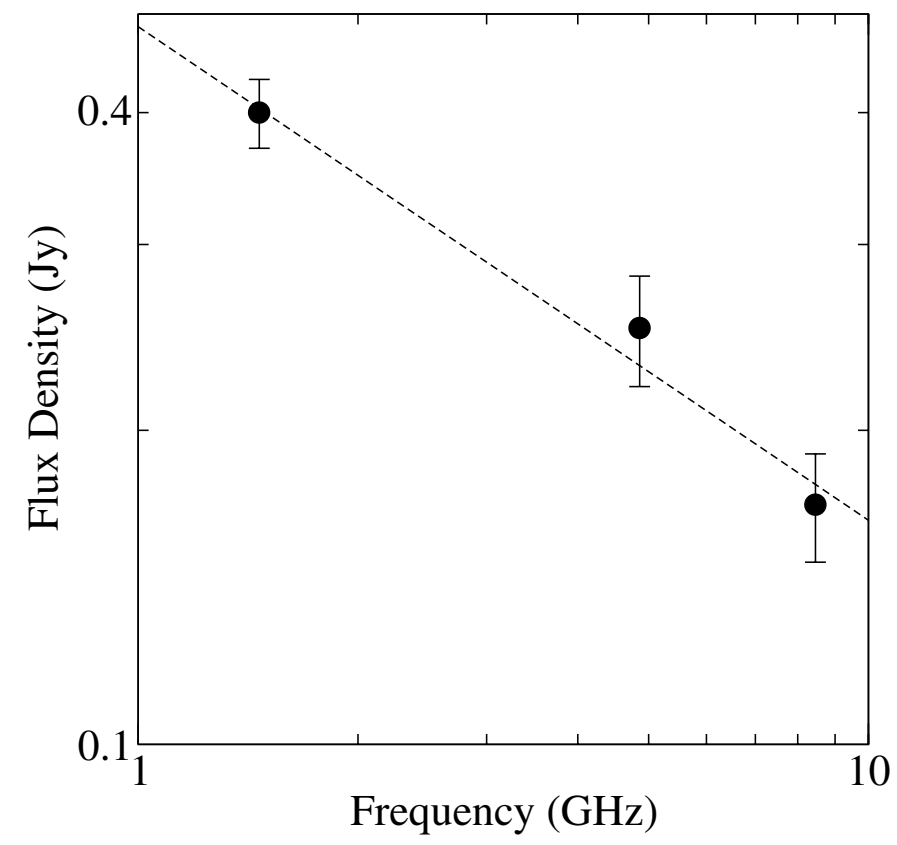

Fig. 5. Radio spectrum of the W43 central cluster based on different sets of VLA observations as explained in the text. The strainght line is a simple power-law fit to the Table 2 flux densities that clearly yields a negative spectral index.

\section{Discussion}

The fact that the WR star W43 \#1 is now a clearly resolved binary confirms the early spectroscopic suspicion of a companion star (Blum et al. 1999), as well as the colliding wind binary scenario recently invoked to interpret the system's X-ray emission (Anderson et al. 2011). The observed close sub-arcsecond angular separation (about 0 .' $^{\prime}$ ) is comparable to other WR binaries resolved by optical telescopes such as the Hubble Space Telescope (e.g. Niemela et al. 1998). We have estimated the probability of a chance alignment to be as low as $~ 5 \%$, based on the average surface star density of the cluster and assuming a Poisson distribution. The expected orbital period is likely to be very long, possibly decades or longer. Indeed, at the assumed W43 large distance the linear separation between components is likely in the range of thousands of astronomical units. The unfortunate fact that colors for the W43 \#1 components cannot be derived from our data owing to saturation problems in the
$H$ - and $K$ s-bands prevents us from any photometric classification. However, W43 \#1b is exactly coincident with the bright X-ray source CXO J184736.6-015633 detected by the Chandra satellite with a $10.8 \sigma$ confidence level (see its error circle in Fig. 3). In a WR binary system, the X-ray emitting region due to colliding winds is normally close to the star with a weaker wind. Therefore, we speculate that W43 \#1a is likely the WR member of the system, while W43 \#1b is its O-type companion. Additional dedicated observations should confirm this point.

It is intriguing that the peak of non-thermal radio emission from the W43 direction does not coincide with the WR system but it is very close to the luminous star W43 \#3 instead, nearly $3^{\prime \prime}$ to the south. This object could also be a binary system, as already mentioned in Sect. 2. Based on the distance and extinction values quoted in the introduction, the colors of the brightest W43 \#3a component derived from our photometric data in Table 1 are consistent with an O-type supergiant. For the W43 \#3b component, only a $K$ s-band absolute magnitude can be derived, which turns out to agree with a main sequence O-type star.

Given the significantly high brightness temperature and negative spectral index observed, the $\mathrm{W} 43$ radio emission is likely to be of non-thermal synchrotron nature. Winds from luminous stellar objects are known to be capable of accelerating relativistic electrons, from which synchrotron emission is naturally expected even if they are single (see e.g. van Loo 2005). However, from the radio spectrum in Sect. 3 the monochromatic $6 \mathrm{~cm}$ luminosity is $2.4 \times 10^{22} \mathrm{erg} \mathrm{s}^{-1} \mathrm{~Hz}^{-1}$, which exceeds the average non-thermal luminosity of a typical WR star (Capman et al. 1999) by nearly three orders of magnitude. This, together with the clearly extended appearance of the radio source, suggests that the collective effect of stellar winds of the WR and O stars in the cluster is the responsible for this non-thermal radio emission. Indeed, the possibility that open clusters may harbor a largescale population of particles up to $\mathrm{TeV}$ energies accelerated by their strongest $\mathrm{O}$ star winds, and even supernova remnants, has already been seriously considered by recent theoretical models (see e.g. Romero 2010). In this context, the detection of nonthermal radio emission and the likely binary nature for the most luminous stars in the cluster give support to the identification of W43 with the HESS J1848-018 gamma-ray source (Chaves et al. 2009).

Assuming standard energy equipartition conditions between the relativistic electrons and the magnetic field (Pacholczyk 1970), the radio source total energy content is estimated to be $2.2 \times 10^{45} \mathrm{erg}$ with a magnetic field of $6.8 \times 10^{-4} \mathrm{G}$. Future theoretical work should ascertain whether this energy content and magnetic field are feasible within the proposed scenario. Alternatively, we cannot exclude the possibility that the detected radio source, whether or not it is related to the high-energy source, is of extragalactic origin. In this case, the overlapping with the cluster itself would be a mere line-of-sight coincidence, but the probability of this being true is negligible. Indeed, based on extragalactic radio-source counts (see e.g. the formulation by Rodríguez et al. 1989), the expected number of radio sources as bright as the one reported here within a solid angle similar to that of the W43 central cluster is $\sim 10^{-6}$.

\section{Conclusions}

The main results of this paper can be summarized as follows:

1. Based on archival data, we have reported the best angular resolution observations of the W43 central cluster at infrared 
and radio wavelengths, which shed some light on its possible association with high-energy gamma-ray emission.

2. The binary nature of the WR star W43 \#1 has been confirmed by resolving its components with sub-arcsecond angular separation. One of them is clearly coincident with the Chandra $\mathrm{X}$-ray source in the field. Another member of the cluster is also proposed to be a binary system consisting of two O-type stars.

3. Non-thermal extended radio emission is clearly detected from the direction of the cluster. We speculate that it could be the result of a collective effect of the stellar winds from the whole population of luminous massive stars in the W43 cluster as the most conceivable scenario. Other alternative interpretations could also be considered and more observations are required to fully solve this issue.

Acknowledgements. The authors acknowledge support of different aspects of this work by grants AYA2010-21782-C03-03 from the Spanish Government, Consejería de Economía, Innovación y Ciencia of Junta de Andalucía as research group FQM-322 and excellence fund FQM-5418, as well as FEDER funds. Based on observations made with the European Southern Observatory telescopes obtained from the ESO/ST-ECF Science Archive Facility. The NRAO is a facility of the NSF operated under cooperative agreement by Associated Universities, Inc. This publication makes use of data products from the Two Micron All Sky Survey, which is a joint project of the University of Massachusetts and the Infrared Processing and Analysis Center/California Institute of Technology, funded by the National Aeronautics and Space Administration and the National Science Foundation in the USA. This research has made use of the NASA/IPAC Infrared Science Archive, which is operated by the Jet Propulsion Laboratory, California Institute of Technology, under contract with the National Aeronautics and Space Administration, and of the Spitzer-GLIMPSE database, which is operated by the Jet Propulsion Laboratory, California Institute of Technology. This research has made use of data obtained from the Chandra Data Archive and the Chandra Source Catalog. Authors also acknowledge useful comments and discussion with Prof. Gustavo E. Romero (IAR).

\section{References}

Abdo, A. A., Ackermann, M., Ajello, M., et al. 2009, ApJS, 183, 46 Abdo, A. A., Ackermann, M., Ajello, M., et al. 2010, ApJS, 188, 405 Aharonian, F., Akhperjanian, A. G., Bazer-Bachi, A. R., et al. 2007, A\&A, 467, 1075

Anderson, G. E., Gaensler, B. M., Kaplan, D. L., et al. 2011, ApJ, 727, 105

Bally, J., Anderson, L. D., Battersby, C., et al. 2010, A\&A, 518, L90

Blum, R. D., Damineli, A., \& Conti, P. S. 1999, AJ, 117, 1392

Chapman, J. M., Leitherer, C., Koribalski, B. 1999, Proc. IAU Symp., 193, 59

Chaves, R. C. G., Renaud, M., Lemoine-Goumard, M., \& Goret, P. 2009, in High Energy Gamma-Ray Astronomy, 4th International Meeting on High Energy Gamma-Ray Astronomy, ed. F. A. Aharonian, W. Hofmann, \& F. M. Rieger, American Institute of Physics, 372

Helfand, D. J., Becker, R. H., White, R. L., Fallon, A., \& Tuttle, S. 2006, AJ, 131,2525

Nguyen Luong, Q., Motte, F., Schuller, F., et al. 2011, A\&A, 529, A41

Niemela, V. S., Shara, M. M., Wallace, D. J., et al. 1998, AJ, 115, 2047

Pacholczyk, A. G. 1970, Radio astrophysics (San Francisco: W. H. Freeman)

Pandian, J. D., Momjian, E., \& Goldsmith, P. F. 2008, A\&A, 486, 191

Rodríguez, L. F., Myers, P. C., Cruz-González, I., \& Terebey, S. 1989, ApJ, 347, 461

Romero, G. E. 2010, Mem. S. A. It., 81, 181

Smith, L. F., Biermann, P., \& Mezger, P. G. 1978, A\&A, 66, 65

Skrutskie, M. F., Cutri, R. M., Stiening, R., et al. 2006, AJ, 131, 1163

van der Hutch, K. A. 2001, New Astron. Rev., 45, 135

Van Loo, S. 2005, Ph.D. Thesis Katholieke Universiteit Leuven, Belgium

Westerhout, G. 1958, Bulletin of the Astronomical Institutes of the Netherlands, 488,215 